\title{
A virtual laboratory for radiotracer and sealed-source applications in industry
}

\author{
Mohammed Siddig H. Mohammed (1), \\ Essam M. Banoqitah (1), \\ Ezzat Elmoujarkach (D), \\ Abdulsalam M. Alhawsawi \\ Fathi Djouider
}

\begin{abstract}
Radioactive sealed sources and radiotracer techniques are used to diagnose industrial process units. This work introduces a workspace to simulate four sealed sources and radiotracer applications, namely, gamma scanning of distillation columns, gamma scanning of pipes, gamma transmission tomography, and radiotracer flow rate measurements. The workspace was created in Geant4 Application for Tomographic Emission (GATE) simulation toolkit and was called Industrial Radioisotope Applications Virtual Laboratory. The flexibility of GATE and the fact that it is an open-source software render it advantageous to radioisotope technology practitioners, educators, and students. The comparison of the simulation results with experimental results that are available in the literature showed the effectiveness of the virtual laboratory.
\end{abstract}

Keywords: Sealed sources • Radiotracers • Industrial gamma tomography • Simulation • GATE

M. S. H. Mohammed ${ }^{\bowtie}$, E. M. Banoqitah,

E. Elmoujarkach, A. M. Alhawsawi, F. Djouider

Department of Nuclear Engineering

King Abdulaziz University

P. O. Box 80204, Jeddah, 21589, Saudi Arabia

E-mail: mshmohamad@kau.edu.sa

Received: 11 May 2020

Accepted: 3 June 2020

\section{Introduction}

Radioisotope techniques have long been used to investigate industrial process units such as distillation columns, pebble bed reactors, fluidized bed, risers, heat exchangers, and process piping. Radioisotope techniques are classified into two categories: sealed sources (gamma scanning and neutron backscattering) and radiotracers (residence time distribution (RTD) measurements, leak detection, mixing efficiency, and flow rate measurements) [1-6]. The advances in the technology afforded industrial process gamma computed tomography (CT) - with its two main modalities gamma transmission tomography and gamma emission tomography - to provide substantial advantages in the diagnostic imaging of multiphase industrial processes [7-9].

Computer simulations can offer advantages to the radioisotope technology used for the analysis of industrial processes. Simulations can be used to assess radiation safety by calculating the radiation dose distribution before performing an investigation, to train practitioners, or to educate students where radioactive materials are not available or an adequate understanding of safe practice is still to be reached. Another valuable utilization of the simulations is that they can be used to understand the expected behaviour of radiation interaction in specific experiments to enhance practice and obtain more reliable results. The simulations give users an understanding regarding how each parameter of the experiment could affect the results, giving them

0029-5922 @ 2021 The Author(s). Published by the Institute of Nuclear Chemistry and Technology.

This is an open access article under the CC BY-NC-ND 4.0 licence (http://creativecommons.org/licences/by-nc-nd/4.0/). 
the ability to optimize the available methods and to evaluate new approaches and techniques.

Monte Carlo codes have been used for gamma scanning of distillation columns to optimize detector models by calculating their efficiencies under different energies and at different distances [10], to design collimators and investigate their effect on the performance of the gamma scanning system [11], and to provide a visual representation of the process and anomalies inside the columns [12].

The evolution of gamma scanning for industrial CT has been associated with the increased use of simulation software for testing image reconstruction algorithms [13], collimator optimization [14], and design of tomographic systems [15].

For the radiotracer techniques, modelling software has been used to process the RTD measurements and analyse the results $[16,17]$. Monte Carlo codes have been used to determine the detection system required to produce the desired single-photon emission CT (SPECT) image resolution [18].

The above literature shows the key additions that the simulation software can offer to the industrial isotope technology. However, these studies have two main disadvantages. First, they have used licensed software, which significantly limits the accessibility and usefulness, and the second is their narrow scope, addressing specific applications, which again limits the value to interested users.

In this work, simulation setups for industrial process investigations using radioisotopes are developed to cover four experiments in one workspace to provide a comprehensive Industrial Radioisotope Applications Virtual Laboratory for students, educators, and technology practitioners. The workspace is developed in the open-source toolkit Geant4 Application for Tomographic Emission (GATE) to enable easier access for users. This work is a novel application of the GATE software toolkit.

\section{Methods}

\section{Evolution of GATE}

GATE is an open-source Monte Carlo simulation toolkit that was developed in 2004 for simulating medical tomographic emission imaging devices [19]. Since then, it has been updated, and new packages have been added to it, increasing its field of applications. GATE uses a macro language to write the codes instead of $\mathrm{C}++$, the build language for Geant4. The macro language makes GATE easier to use and requires no knowledge of $\mathrm{C}++$. The current use of GATE is in the medical imaging field (using both external and internal sources) [20,21], dosimetry [22-24], radiotherapy (using photons and charged particles), and the newly added feature of optical imaging.

To define a material in GATE, a database file is supplied by GATE containing the most used elements and materials. This file contains all the information required for GATE to assign the nuclear properties from the Geant4 data sets. Moreover, the user can add any element or material into the simulation by supplying specific information regarding the material. There are two methods to add new materials or mixtures into the simulation: using the chemical formula of the material or defining the fraction of each element or mixture [25]. GATE can read and build geometry from stereolithography file formats, which would be critical when dealing with complex geometries.

To define a radioactive source using GATE, there are a few properties that a user can control: the volume of the source, the location where it is placed within the simulation world, the energy (it can be either discrete energy or a spectrum), particle type (gamma rays, neutrons, or electrons), the activity or number of primaries, and the solid angle to transform the source from an isotropic one into a directional beam. The user also can use a predefined source extracted from Geant4 database.

The physics list is the library that the simulation toolkit uses to calculate and track the interaction of radiation with matter. To simulate the interaction of electromagnetic particles with matter, the physics list's electromagnetic processes are used. The mean free path of a process, $\lambda$, is given in terms of the total cross section as follows:

$$
\lambda(E)=\left(\sum_{i}\left[n_{i} \cdot \sigma\left(Z_{i}, E\right)\right]\right)^{-1}
$$

where $\sigma\left(Z_{i}, E\right)$ is the cross section of the process of atoms composing the material. During the start-up of the simulation, the values of the cross sections per atom and the mean free path values are extracted from the simulation database and used [25].

The outputs of GATE can be in various formats depending on the method of saving the output using a sensitive detector or an actor. In the virtual laboratory introduced in this paper, three types of outputs were used, namely, files with ".sin", ".dat", and ".txt" extensions. Files with ".sin" extension are images that can be read using the ImageJ toolkit (supplied with vGate or can be installed by visiting the Image J website) or other image-processing toolkits.

Using the virtual laboratory does not require working knowledge of GATE. The user can either choose commands from a defined list (to describe the experiment's geometry and materials, physics models, radiation detectors, and radiation source) or execute a macro file that contains a patch of commands.

\section{Gamma scanning of distillation columns and pipes}

Gamma ray scanning is used to conduct an internal investigation of distillation columns and pipes while the equipment is in operation. A radioactive source is placed in contact with the exterior of the column or the pipe investigated, to emit gamma rays that penetrate through the diameter of the object. The gamma ray intensity is modified by the densities of the materials of the equipment's internals. A radiation detector is placed in the opposite direction, which is usually used in the pulse mode to 
be able to resolve the primary photons, scattered radiation, and background radiation. The source and the detector are moved along the equipment's exterior simultaneously. The measured gamma ray intensity, which is described by Eq. (2), is plotted to provide a density profile.

$$
I=I_{\mathrm{o}} \cdot \exp \cdot(-\mu \rho x)
$$

where $I$ is the intensity of radiation transmitted through the material, $I_{0}$ is the intensity of incident radiation, $\mu$ is the absorption coefficient of the material measured, $\rho$ is the material's density, and $x$ is the material's thickness.

The estimated strength of the source can be calculated as follows:

$$
\text { Activity }(\mathrm{mCi})=\frac{D d^{2} 2^{\frac{e}{X_{1 / 2}}}}{\Gamma}
$$

where $D$ - dose rate required at the detector (practically accepted as $10 \mu \mathrm{Sv} / \mathrm{h}$ ), $d$ - internal diameter of the column $(\mathrm{m}), e$ - double wall thickness of the column $(\mathrm{mm}), X_{1 / 2}$ - half-value thickness $(\mathrm{mm})$ for steel and the source (e.g., $22 \mathrm{~mm}$ for steel and Co-60), $\Gamma$-gamma constant for the gamma source (e.g., $13.5 \mathrm{mSv} / \mathrm{h}$ for $1-\mathrm{m}$ distance in air for Co-60 source of $1 \mathrm{Ci}$ ).

The objective of the distillation column scanning is to investigate the integrity of the column trays and characterize the liquid and vapour phases.

The column was designed using SolidWorks ${ }^{\mathrm{TM}}$ to make the simulation of gamma ray scanning as accurate as in practical work. SolidWorks ${ }^{\mathrm{TM}}$ is a computer-aided design (CAD) program that can be used to build complicated objects. Afterward, the object can be exported in a Standard Tessellation Language (STL) file format. GATE v8.0 and newer versions support this format. STL files can be read and imported into the simulation, allowing the use of any complicated geometry with very high accuracy. The column was designed to have a $25-\mathrm{cm}$ diameter to reduce the computation time, and its material was low-carbon steel. The radioactive source was defined as ${ }^{60} \mathrm{Co}$ with energies of $1.1 \mathrm{MeV}$ and $1.3 \mathrm{MeV}$. The radiation detector was set as a sodium iodide ( $\mathrm{NaI}(\mathrm{Tl})$ ) detector. A view of the longitudinal section of the design drawing is shown in Fig. 1.

The simulation setup showing the column, collimated ${ }^{60} \mathrm{Co}$ source, and radiation detector is given in Fig. 2. The parameters that the user can control in the simulation are the source type, the material of the column wall, the diameter of the column, the detector crystal, damaged or intact column internals, and the step of the scan.

\section{Pipe scanning}

The scanning of pipelines and flow lines is used for detecting the sludging effects such as blockages and volume narrowing; furthermore, it can be used to indicate the presence of vapour and liquid phases and the integrity of the pipe's material. It is an

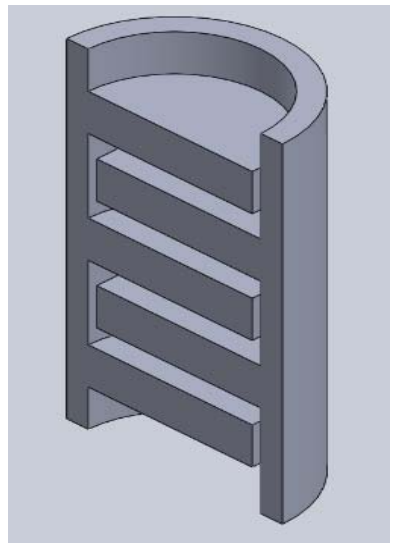

Fig. 1. Section of the distillation column that was designed using SolidWorks ${ }^{\mathrm{TM}}$.

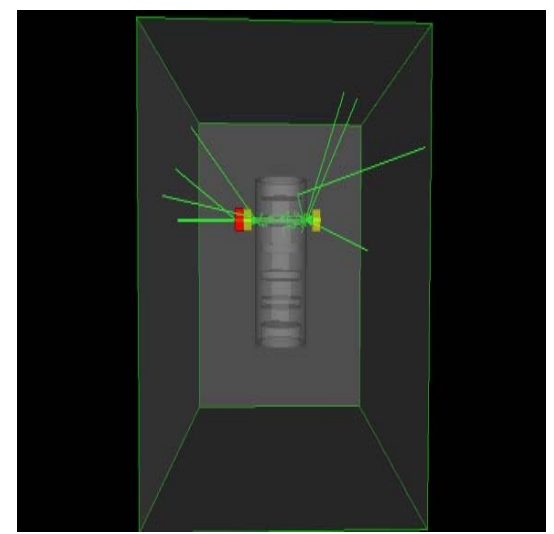

Fig. 2. The simulation setup of the column scan.

advantageous technique because it is fast, does not interrupt the pipe's process, and uses low-strength gamma sources.

For the simulation of this technique, the pipe was designed using SolidWorks ${ }^{\mathrm{TM}}$ with an option to add deposited material in the inner surface. Figure 3 shows the used pipe scanning setup, which simulates the arrangement of the experimental technique. In addition to radiation source and detector, the user can change the material and dimension of the pipe and the density of the residue material. The material of the pipe used in this work was low-carbon steel, and its diameter and thickness were $20 \mathrm{~cm}$ and $10 \mathrm{~mm}$, respectively. The gamma ray source was defined as ${ }^{137} \mathrm{Cs}$ with energy of $660 \mathrm{keV}$, the detector
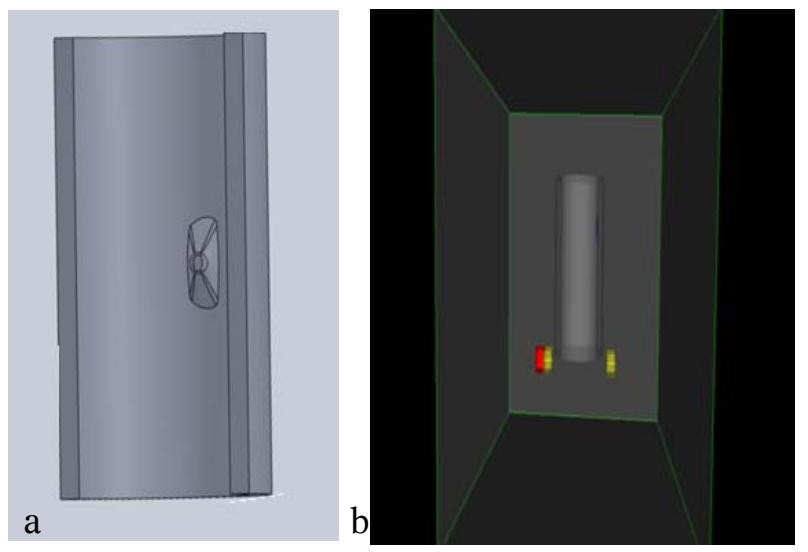

Fig. 3. (a) SolidWorks ${ }^{\mathrm{TM}}$ design of the pipe with solid deposit. (b) Simulation setup. 


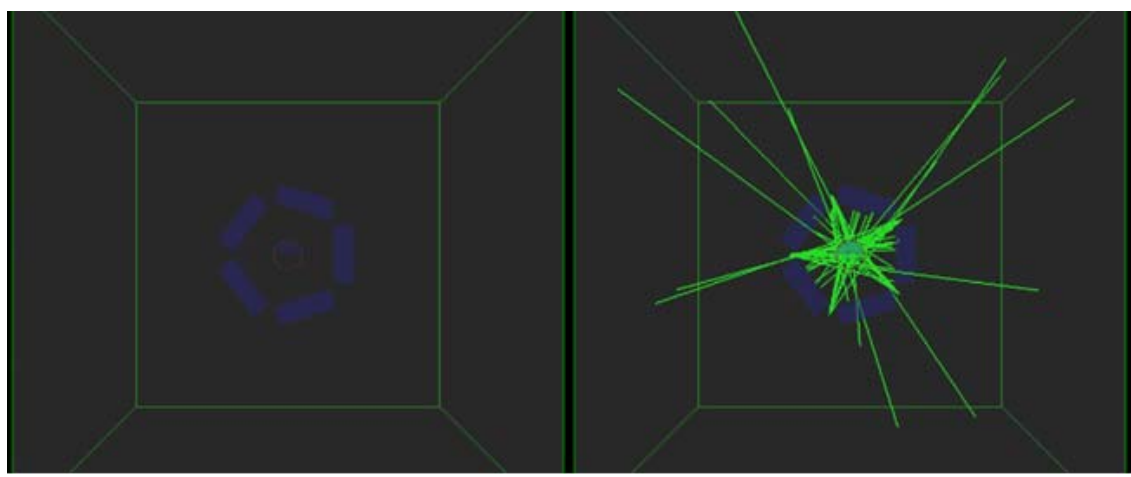

Fig. 4. Simulation setup for the fifth-generation CT scan.

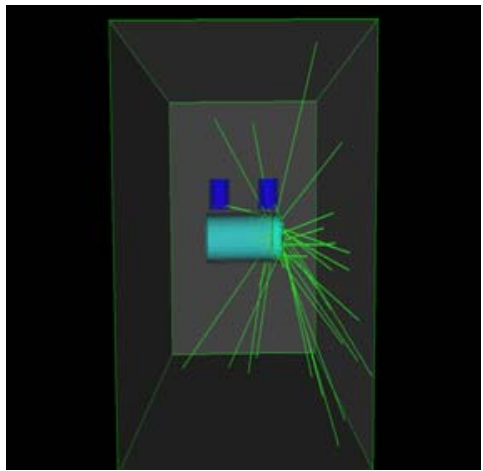

Fig. 5. The simulation setup used for flow rate measurement using a radiotracer.

was $\mathrm{NaI}(\mathrm{Tl})$, and the residue was chosen to imitate mud with a density of $1.9 \mathrm{~g} / \mathrm{cm}^{3}$ and composed of hydrogen, oxygen, and silicon.

\section{Gamma transmission tomography}

Multiphase equipment containing gas-liquid, liquid-solid, gas-solid, gas-liquid-solid, and liquid-liquid-solid-gas are important components in major industries, such as petrochemical, minerals, pharmaceuticals, and food.

Gamma transmission CT is used for the imaging of multiphase systems to measure and quantify the phase distributions. The CT system uses several detectors to provide multiple measurements that can be used to reconstruct cross-sectional information of the system's phases.

The third simulation in the virtual laboratory was for the CT of multiphase flow in a low-carbon steel pipe. A fifth-generation industrial CT scanning system was used, where five detectors were placed around the pipe with five stationary fixed sources.

Figure 4 depicts the simulation setup used to realize the CT system. The user can vary the radioisotope, detectors, pipe's material and dimensions, scanning step size, and the multiphase flow densities. The simulation was performed modelling a pipe carrying liquid and vapour phases, with a deposited solid phase.

\section{Flow rate measurement}

Radiotracers can be used to accurately measure the flow rate in the process equipment. The radiotracer method can be used to calibrate the conventional flow meters. A radiotracer is injected into the pipe, and two detectors are placed on the outer surface of the pipe at a given distance apart. As the fluid with the radiotracer passes through the pipe, the first detector collects counts, while the other detector receives little-to-no signal. When the radiotracer-labelled fluid moves toward the second detector, the signal from the first detector weakens, while that of the second detector rises. By measuring the time interval between the two signals, the flow rate can be calculated. In this paper, the fluid was set to water and the $140-\mathrm{keV}$ gamma emitter ${ }^{99 \mathrm{~m}} \mathrm{Tc}$ was defined to simulate the radiotracer. Figure 5 shows the setup for simulating flow rate measurement.

\section{Results and discussion}

\section{Results of gamma scanning of distillation columns and pipe}

Figure 6 shows a plot of the gamma ray intensities, which corresponds to the densities of the materials of the column internals. The low gamma ray intensity regions indicate attenuation by the steel trays, and the high-intensity portions indicate the spaces between

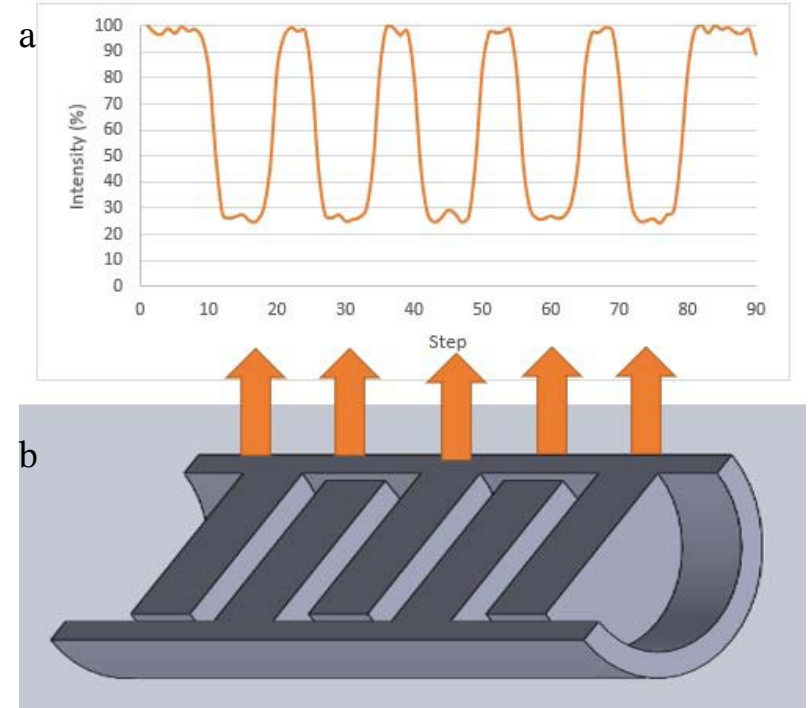

Fig. 6. (a) Simulation results of the gamma ray scanning of a distillation column. (b) A section of a distillation column; the arrows indicate the corresponding intensity measured after radiation has passed through the trays. 


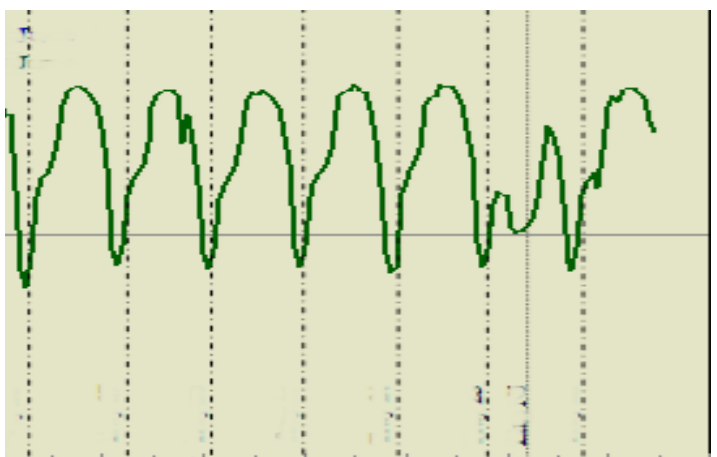

Fig. 7. Experimental gamma scanning profile (reproduced from [26]).

the trays. Figure 7, reproduced from [26], is a part of an experimental density profile obtained from a distillation column scan in an oil refinery using $\mathrm{NaI}(\mathrm{Tl})$ detector and ${ }^{60} \mathrm{Co}$ having an activity of $50 \mathrm{mCi}$. The comparison between Figs. 6 and 7 demonstrates that the simulation result is credible.

If the pattern of the high gamma ray intensities is changed, it would mean an anomaly caused by a high-density material such as a collapsed tray or liquid buildup. Figure 8 a shows the results of scanning a column with a collapsed tray, which means that the space between Trays 2 and 3 is packed with the metal (Fig. 8b), increasing the area in which gamma ray is attenuated (Steps 30-43).

This simulation can be supplemented with models for liquid and vapour phases and various scenarios of process faults.

Figure 9 shows the result of the pipe scan simulation, where the material inside the pipe is distributed homogeneously, resulting in a continuous line. Figure 10 shows the density profile when a solid phase is deposited to disturb or block the flow. The decrease in the intensity between the slices 7 and 10

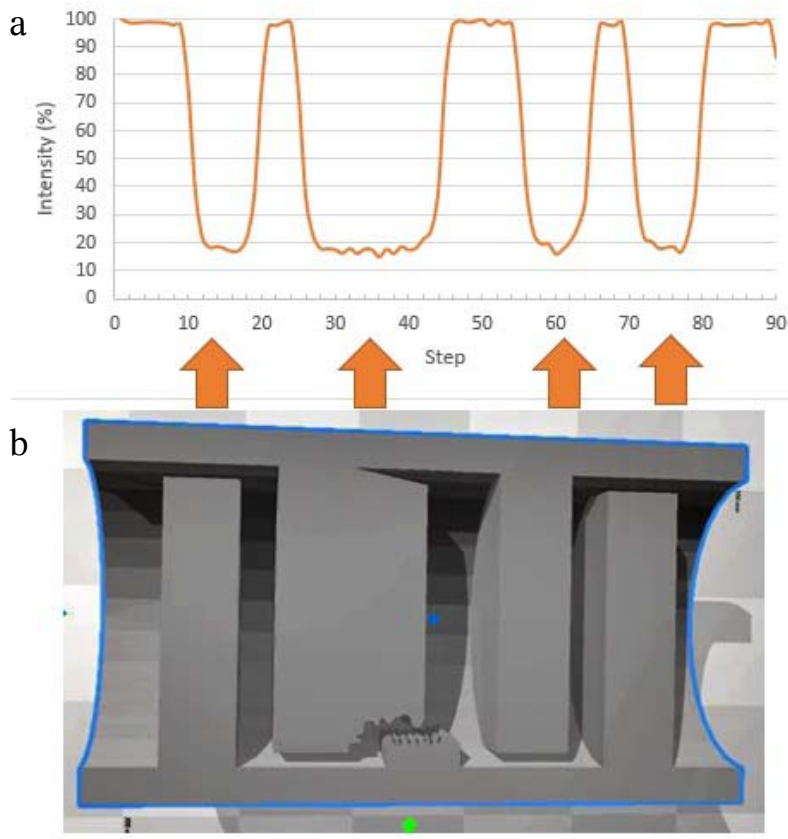

Fig. 8. (a) Simulation results of the gamma ray scanning of a distillation column with a collapsed tray. (b) A section of a distillation column with a collapsed tray.

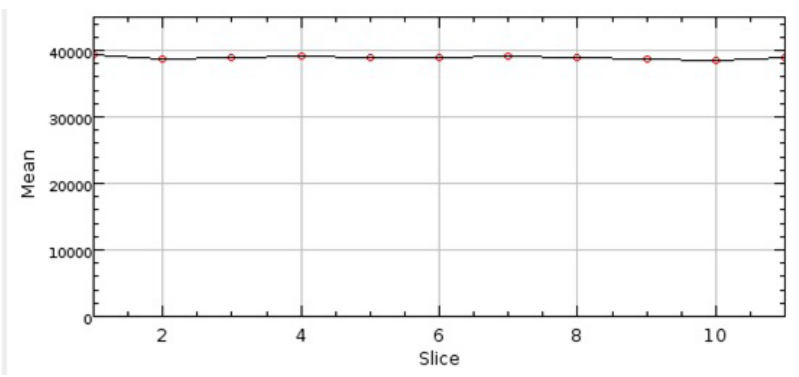

Fig. 9. Simulation results of gamma scanning of a deposit-free pipe.

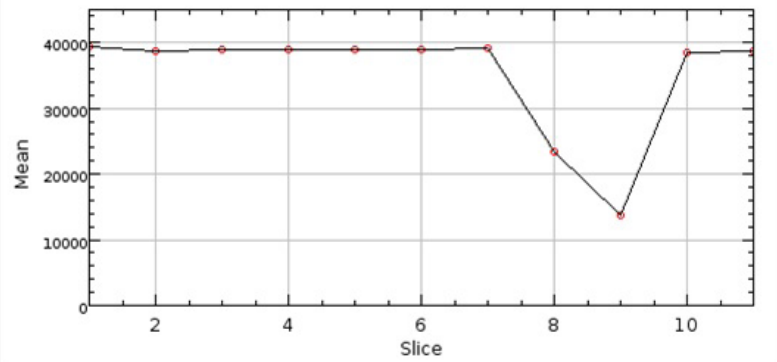

Fig. 10. Simulation results of a pipe with deposited solid phase.
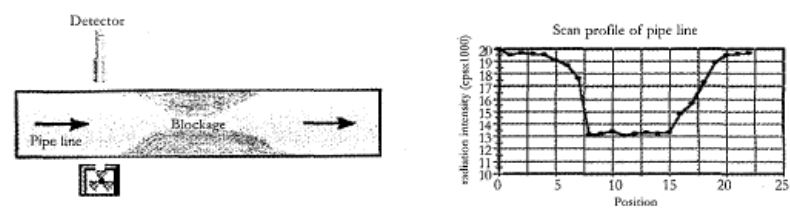

Fig. 11. Application of gamma scanning to detect blockage in pipes (reproduced from [27]).

can be used to describe the deposits' location, density, and area. Figure 11 illustrates the application of gamma scanning for the detection of blockages in pipes. The comparison of Fig. 11 with Fig. 10 shows that the virtual laboratory offers adequate results in this application.

\section{Results of gamma transmission tomography}

The CT output is 128 pixels $\times 128$ pixels and the number of images depends on the number and size of the steps used in the simulation multiplied by the number of cameras used. Five cameras were used with seven steps, resulting in 35 images.

For the pipe CT, Fig. 12 shows projections obtained from two detectors shown in a grayscale, where white represents the highest radiation intensity and black represents the lowest intensity. Figure 12a shows the liquid phase in black because it significantly attenuated the radiation intensity, compared to the gas phase (air) shown in gray because the radiation was slightly attenuated mainly due to the distance effect. Figure 12b shows the deposited solid phase as a half-sphere.

GATE does not permit image reconstruction, so the user needs to export the obtained projections to other software toolkits that allow the use or build of reconstruction algorithms. 

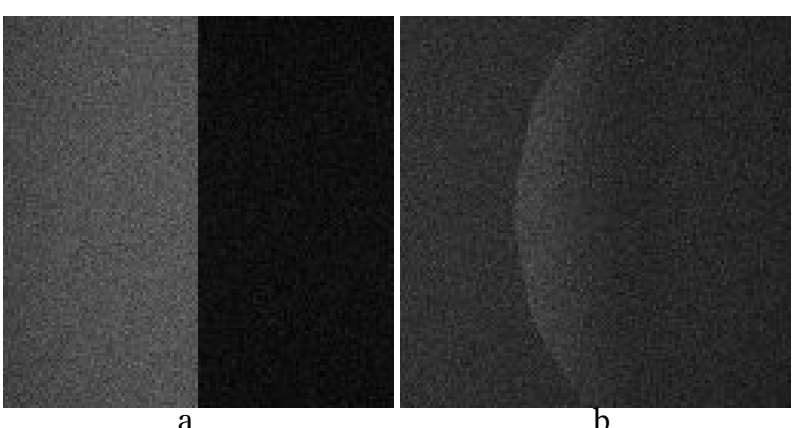

a

b

Fig. 12. The five projections of CT of the pipe.

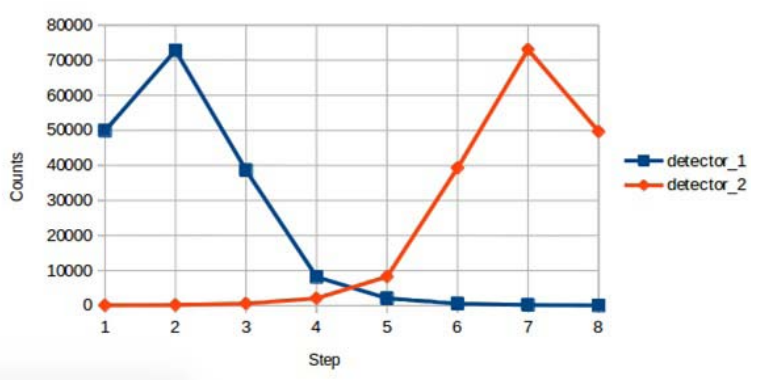

Fig. 13. Simulation result of the flow rate measurement.

\section{Results of radiotracer flow rate measurement}

Figure 13 shows the output obtained when running the flow rate simulation. The two peaks are the responses of the detectors to the passage of tracer. The positions of the detectors and the times when the peaks were detected are used to calculate the flow rate.

In this simulation, the pipe diameter was $0.165 \mathrm{~m}$, the distance between the two detectors was $0.26 \mathrm{~m}$, and the time between the two peaks was $5 \mathrm{~s}$. The flow rate $Q$ is calculated as follows:

$$
Q=A \times V
$$

where $A$ is the cross-sectional area and $V$ is the liquid's velocity.

Therefore, $Q=0.0191 \times 0.052=0.00099 \mathrm{~m}^{3} \cdot \mathrm{s}$.

\section{Conclusion}

The virtual laboratory created in GATE toolkit for the simulation of radioisotope applications used in diagnosing and troubleshooting industrial process equipment included four important applications: distillation column scanning, pipe scanning, gamma transmission tomography, and flow rate measurement. The simulations gave results comparable to those obtained in the practical experiments. However, the capabilities of GATE to track a liquid flow labelled with radioactive material is limited; so flow measurement was the only radiotracer experiment that could be simulated.

The virtual laboratory is meant to be user-friendly, offering options to edit various parameters of the experiments to allow examination of different scenarios.
The virtual laboratory was introduced to the researchers and educators at the Department of Nuclear Engineering of King Abdulaziz University to apply it in their activities and provide feedback on the required improvements.

Optimizations to the simulations and experimental validation are considered in ongoing work; furthermore, other radioisotope applications will be added.

Acknowledgment. This work was supported by the Deanship of Scientific Research (DSR), King Abdulaziz University, Jeddah, under grant no. (G:1296-135-1440). The authors, therefore, gratefully acknowledge the DSR technical and financial support.

\section{ORCID}

A. M. Alhawsawi (D) http://orcid.org/0000-0002-7992-8850 E. M. Banoqitah (1) http://orcid.org/0000-0002-9127-6322 F. Djouider (ID http://orcid.org/0000-0003-3445-816X E. Elmoujarkach (D) http://orcid.org/0000-0003-2750-5984 M. S. H. Mohammed (DD http://orcid.org/0000-0002-4238-9566

\section{References}

1. Mohd Yunos, M. A. S., Hussain, S. A., Hamdan, M. Y., \& Jaafar, A. (2016). Industrial radiotracer technology for process optimizations in chemical industries - A review. Pertanika Journal of Scholarly Research Reviews, 2(3), 20-46.

2. Charlton, J. S. (1986). Radioisotopes in industry. In J. S. Charlton (Ed.), Radioisotope techniques for problem-solving in industrial process plants (pp. 1-8). Dordrecht: Springer. https://doi.org/10.1007/97894-009-4073-4_1.

3. International Atomic Energy Agency. (2004). Radiotracer applications in industry - a guidebook. Vienna: IAEA. (Technical Report Series no. 423). Available from https://www-pub.iaea.org/MTCD/Publications/ PDF/TRS423_web.pdf.

4. Jin, J. -H., \& Thereska, J. (2004). Industrial applications of radiotracer and sealed source technology promoted by IAEA. In: Tracer 3. International Conference on Tracers and Tracing Methods, 22-24 June 2004, Ciechocinek, Poland.

5. Farooq, M., Khan, I. H., Ghiyas-ud-Din, , Gul, S., Palige, J., \& Chmielewski, A. G. (2003). Radiotracer investigations of municipal sewage treatment stations. Nukleonika, 48(1), 57-61.

6. Smolinski, T., Rogowski, M., Brykala, M., Pyszynska, M., \& Chmielewski, A. G. (2018). Studies on hydrometallurgical processes using nuclear techniques to be applied in copper industry. I. Application of ${ }^{64} \mathrm{Cu}$ radiotracer for investigation of copper ore leaching. Nukleonika, 63(4), 123-129. https://doi.org/10.2478/ nuka-2018-0015.

7. International Atomic Energy Agency. (2008). Industrial process gamma tomography. Vienna: IAEA. (IAEATECDOC-1589). Available from https://www-pub. iaea.org/MTCD/Publications/PDF/TE_1589_web.pdf. 
8. Wang, M. (Ed.). (2015). Industrial tomography: Systems and applications. Elsevier. https://doi. org/10.1016/C2013-0-16466-5.

9. Calvo, W. A. P., Hamada, M. M., Sprenger, F. E., Vasquez, P. A. S., Rela, P. R., Martins, J. F. T., de Matos Pereira, J. C. S., Omi, N. M., \& de Mesquita, C. H. (2009). Gamma-ray computed tomography SCANNERS for applications in multiphase system COLUMNs. Nukleonika, 54(2), 129-133.

10. Chuong, H. D., Hung, N. Q., My Le, N. T., Nguyen, V. H., \& Thanh, T. T. (2019). Validation of gamma scanning method for optimizing $\mathrm{NaI}(\mathrm{Tl})$ detector model in Monte Carlo simulation. Appl. Radiat. Isot., 149, 1-8. https://doi.org/10.1016/j.apradiso.2019.04.009.

11. Shahabinejad, H., \& Feghhi, S. A. H. (2015). Design, optimization and performance of source and detector collimators for gamma-ray scanning of a lab-scale distillation column. Appl. Radiat. Isot., 99, 25-34. https://doi.org/10.1016/j.apradiso.2015.02.008.

12. Zain, R. M., Yahya, R., \& Mahmood, A. A. (2009). Simulation of gamma scan study on column test rig at evaluation and verification Nuclear Malaysia facility. In Nuclear Malaysia Technical Convention, 6-8 October 2009, Bangi, Malaysia.

13. Haraguchi, M. I., Calvo, W. A. P., \& Kim, H. Y. (2018). Tomographic 2-D gamma scanning for industrial process troubleshooting. Flow Meas. Instrum., 62, 235-245. https://doi.org/10.1016/j.flowmeasinst.2017.09.004.

14. Zhang, J., Tuo, X., Wang, Q., Leng, Y., \& Shi, R. (2018). Monte Carlo simulation and collimator optimization for tomographic gamma scanning. In 2017 IEEE Nuclear Science Symposium and Medical Imaging Conference, NSS/MIC 2017 - Conference Proceedings. Institute of Electrical and Electronics Engineers Inc. https://doi.org/10.1109/NSSMIC.2017.8532877.

15. Kim, J., Jung, S., Moon, J., Kwon, T., \& Cho, G. (2011). Monte Carlo simulation for the design of industrial gamma-ray transmission tomography. Progress in Nuclear Science and Technology, 1, 263-266. Retrieved from http://www.aesj.net/document/pnst001/263.pdf.

16. Kasban, H., Zahran, O., Arafa, H., El-Kordy, M., Elaraby, S. M. S., \& Abd El-Samie, F. E. (2010). Laboratory experiments and modeling for industrial radiotracer applications. Appl. Radiat. Isot., 68(6), 1049-1056. https://doi.org/10.1016/j.apradiso.2010.01.044.

17. Sheoran, M., Chandra, A., Bhunia, H., Bajpai, P., \& Pant, H. J. (2018). Residence time distribution studies using radiotracers in chemical industry-A review. Chem. Eng. Commun., 205(6), 739-758. https://doi. org/10.1080/00986445.2017.1410478.

18. Park, J. G., Kim, C. H., Han, M. C., Jung, S. H., Kim, J. B., \& Moon, J. (2012). Optimization of detection geometry for industrial SPECT by Monte Carlo simulations. J. Instrum., 8, C04006(5pp.). https:// doi.org/10.1088/1748-0221/8/04/C04006.

19. International OpenGATE collaboration. (2020). GATE. Retrieved from http://www.opengatecollaboration.org/

20. Banoqitah, E., Taha, E., Elmoujarkach, E., Alsebaie, S., Subahi, A., \& Alsharif, S. (2018). A Monte Carlo study of arms effect in myocardial perfusion of normal and abnormal cases utilizing STL heart shape. Results Phys., 10, 323-331. https://doi.org/10.1016/j. rinp.2018.06.028.

21. Lee, S., Gregor, J., \& Osborne, D. (2013). Development and validation of a complete GATE model of the Siemens Inveon trimodal imaging platform. Mol. Imaging, 12(7), 434-445. https://doi. org/10.2310/7290.2013.00058.

22. Bouzid, D., Bert, J., Dupre, P. F., Benhalouche, S., Pradier, O., Boussion, N., \& Visvikis, D. (2015). Monte-Carlo dosimetry for intraoperative radiotherapy using a low energy x-ray source. Acta Oncol., 54(10), 1788-1795. https://doi.org/10.3109/028418 6X.2015.1016623.

23. Spirou, S. V., Makris, D., \& Loudos, G. (2015). Does the setup of Monte Carlo simulations influence the calculated properties and effect of gold nanoparticles in radiation therapy? Phys. Medica, 31(7), 817-821. https://doi.org/10.1016/j.ejmp.2015.05.008.

24. Taha, E., Djouider, F., \& Banoqitah, E. (2018). Monte Carlo simulations for dose enhancement in cancer treatment using bismuth oxide nanoparticles implanted in brain soft tissue. Australas. Phys. Eng. Sci. Med., 1-8. https://doi.org/10.1007/s13246-018-0633-z.

25. Jan, S., Santin, G., Strul, D., Staelens, S., Assié, K., Autret, D., Avner, S., Barbier, R., Bardies, M., Bloomfield, P. M., Brasse, D., Breton, V., Bruyndonckx, P., Buvat, I., Chatziioannoull, A. F., Choil, Y., Chung, Y. H., Comtat, D., Donnarieix, D., Ferrer, L., Gllick, S. J., Groissellle, C. J., Guez, D., Honore, P. -F., Kerhoas-Cavata, S., Kirov, A. S., Kohlil, V., Koole, M., Krieguer, M., van der Laan, D. L., Lamare, E., Largeron, G., Lartizien, C., Lazaro, D., Maas, M. C., Maigne, L., Mayet, F., Melot, F., Merheb, C., Pennacchio, E., Perez, J., Pietrzyk, U., Rannoull, F. R., Rey, M., Schaart, D. R., Schmidtlein, C. R., Simon, L., Song, T. Y., Vieira, J. -M., Visvikis, D., Van de Walle, R., Wieers, E., \& Morel, C. (2004). GATE: a simulation toolkit for PET and SPECT. Phys. Med. Biol., 49(19), 4543-4561. https://doi.org/10.1088/00319155/49/19/007.

26. Mohammed, M. S. H. (2007). Investigation of process equipment in petrochemical industry using radioisotope technology. Sudan Academy of Sciences.

27. International Atomic Energy Agency. (2002). Radioisotope applications for troubleshooting and optimizing industrial processes. Vienna: IAEA. 